\title{
Joint Assessment of Structural, Perfusion, and Diffusion MRI in Alzheimer's Disease and Frontotemporal Dementia
}

\author{
Yu Zhang, ${ }^{1,2}$ Norbert Schuff, ${ }^{1,2}$ Christopher Ching, ${ }^{1,2}$ Duygu Tosun,, 2 Wang Zhan,, 2 \\ Marzieh Nezamzadeh, ${ }^{1,2}$ Howard J. Rosen, ${ }^{3}$ Joel H. Kramer, ${ }^{3}$ Maria Luisa Gorno-Tempini, ${ }^{3}$ \\ Bruce L. Miller, ${ }^{3}$ and Michael W. Weiner ${ }^{1,2,3}$ \\ ${ }^{1}$ Center for Imaging of Neurodegenerative Diseases, Department of Veterans Affairs San Francisco VA, Medical Center, \\ 4150, Clement Street, San Francisco, CA 94121, USA \\ ${ }^{2}$ Department of Radiology, University of California, San Francisco, CA 94143, USA \\ ${ }^{3}$ Department of Neurology, University of California, San Francisco, CA 94143, USA
}

Correspondence should be addressed to Yu Zhang, yu.zhang@ucsf.edu

Received 29 November 2010; Accepted 26 April 2011

Academic Editor: Katsuya Urakami

Copyright (c) 2011 Yu Zhang et al. This is an open access article distributed under the Creative Commons Attribution License, which permits unrestricted use, distribution, and reproduction in any medium, provided the original work is properly cited.

\begin{abstract}
Most MRI studies of Alzheimer's disease (AD) and frontotemporal dementia (FTD) have assessed structural, perfusion and diffusion abnormalities separately while ignoring the relationships across imaging modalities. This paper aimed to assess brain gray (GM) and white matter (WM) abnormalities jointly to elucidate differences in abnormal MRI patterns between the diseases. Twenty AD, 20 FTD patients, and 21 healthy control subjects were imaged using a 4 Tesla MRI. GM loss and GM hypoperfusion were measured using high-resolution T1 and arterial spin labeling MRI (ASL-MRI). WM degradation was measured with diffusion tensor imaging (DTI). Using a new analytical approach, the study found greater WM degenerations in FTD than AD at mild abnormality levels. Furthermore, the GM loss and WM degeneration exceeded the reduced perfusion in FTD whereas, in AD, structural and functional damages were similar. Joint assessments of multimodal MRI have potential value to provide new imaging markers for improved differential diagnoses between FTD and AD.
\end{abstract}

\section{Introduction}

Alzheimer's disease $(\mathrm{AD})$ and frontotemporal dementia (FTD) are two of the most common and devastating disorders that result in dementia in the elderly population. Although the definitive diagnosis of each type of dementia is not possible until autopsy, biomarkers based on magnetic resonance imaging (MRI), providing measurements of brain volume, perfusion, and white matter integrity, have been promising for improved diagnosis and prediction of dementia progression [1]. In $\mathrm{AD}$, which is a progressive dementing disorder associated with cognitive impairments beginning with episodic memory deficits, MRI measurements of brain volume have shown characteristic gray matter (GM) loss primarily in medial temporal lobe regions $[2,3]$ whereas functional studies using arterial spin labeling MRI or PET/SPECT imaging have shown prominent changes primarily in the parietal lobe (including the posterior cingulate gyrus and lat- eral temporoparietal areas) [4-7], though regions of structural, and functional alterations can overlap. Furthermore, diffusion tensor imaging (DTI), a unique method to assess the integrity of white matter microstructure, have revealed white matter (WM) alterations in $\mathrm{AD}$, involving the parietal, temporal, and frontal brain regions [8-13]. In FTD, which is associated with impairments of social behaviors, personality, and executive functions, MRI has shown characteristic patterns of structural GM loss $[14,15]$ and GM dysfunction [16-20] primarily in frontal and anterior temporal lobes. WM volume loss $[21,22]$ and WM degradation in FTD [23-25] have also been reported in the frontal and temporal regions.

Using biomarkers of neurocognitive measurements to differentiate between $\mathrm{AD}$ and FTD are often difficult because of overlapping symptoms. Several studies using imaging markers compared differences in abnormal brain patterns between AD and FTD directly. Structural MRI showed that 
$\mathrm{AD}$ was associated with greater GM loss than FTD in posterior brain regions $[26,27]$ whereas FTD was associated with more severe GM loss than was AD in frontal brain regions [26, 28-30]. Similarly, functional imaging such as PET/SPECT and perfusion MRI showed that AD was associated with greater reduced cerebral blood flow or metabolism than FTD in parietal and occipital brain regions $[16,28$, 30-32]; whereas FTD was associated with greater frontal dysfunction than $\mathrm{AD}[16,30,32]$. In addition to differences in GM, we have previously reported differences in WM between AD and FTD [25]. Specifically, measurements of fractional anisotropy (FA) — a summary measure of DTI indexing WM integrity-indicated greater WM degradation (FA reduction) of frontal brain regions in FTD compared to $\mathrm{AD}$. Furthermore, no brain region in $\mathrm{AD}$ was shown to have more WM degradation when compared to FTD. Taken together, these findings suggest that AD and FTD are each associated with disease-specific regional patterns of GM and WM alterations. Recent multimodality strategies [33, 34] of combined radiological markers such as analyzing brain volume and perfusion or WM changes together have shown superior power than that using conventional single modality domain (e.g., brain volume measurement alone) in diagnosis of $\mathrm{AD}$. However, to our knowledge, rarely did MRI studies evaluate GM and WM differences between AD and FTD jointly.

In this study we present a new approach to compare structural, perfusion, and diffusion alterations between $\mathrm{AD}$ and FTD using T1-weighted high-resolution structural MRI, arterial spin-labeled perfusion MRI (ASL-MRI), and DTI. The objective was to determine if a joint evaluation of multimodal MRI could provide a biomarker for a differential diagnosis between $\mathrm{AD}$ and FTD.

\section{Subjects and Methods}

2.1. Subjects. Twenty AD patients (mean age and standard deviation: 63.1 \pm 6.9 yrs) with a Mini-Mental State Examination (MMSE) [35] score of on average 21.9 $\pm 5.6,20$ patients with FTD (age: $60.7 \pm 9.9$ yrs; MMSE: $23.1 \pm 5.6$ ) and 21 cognitively normal $(\mathrm{CN})$ subjects (age: $61.9 \pm 9.6$ yrs; MMSE: $29.6 \pm 0.5$ ) were included in this cross-sectional MRI study. A summary of the subject demographics and relevant clinical information are listed in Table 1. The patients with FTD and $\mathrm{AD}$ were recruited from the Memory and Aging Center of the University of California, San Francisco. All patients were diagnosed based on information obtained from an extensive clinical history and physical examination. The MR images were used to rule out other major neuropathologies such as tumors, strokes, or inflammation but not to diagnose dementia. The subjects were included in the study if they were between 30-80 years old and without history of brain trauma, brain tumor, stroke, epilepsy, alcoholism, psychiatric illness, or other systemic diseases that affect brain function. FTD was diagnosed according to the consensus criteria established by Neary et al. [36]. All FTD patients were diagnosed with the frontal variant subtype, two of which had combined motor neuron-related symptoms. AD patients were diagnosed according to the criteria of the
National Institute of Neurological and Communicative Disorders and Stroke-Alzheimer's Disease and Related Disorders Association (NINCDS/ADRDA) [37]. All subjects received a standard battery of neuropsychological tests including assessment of global cognitive impairment using MMSE and global functional impairment using the Clinical Dementia Rating (CDR) scale [38]. Fifty-seven out of all 61subjects had blood drawn for APOE genotyping. Reliable information about the age of onset of symptoms was available from 12 out of $20 \mathrm{AD}$ patients and from all $20 \mathrm{FTD}$ patients. Because it is not unusual for subjects in this age group to have WM signal hyperintensities (WMSH) on MRI, subjects with WMSH were included. An experienced radiologist reviewed all MRI data, and the scores of WMSH were used as covariates in the analysis. The severity of WMSH was classified as mild (deep white matter lesions $\leq 3 \mathrm{~mm}$, and periventricular hyperintensities $<5 \mathrm{~mm}$ thickness), moderate (deep white matter lesions between 4-10 $\mathrm{mm}$, or periventricular hyperintensities between 6-10 mm thickness), or severe (deep white matter lesions $>10 \mathrm{~mm}$, or periventricular hyperintensities $>10 \mathrm{~mm}$ thickness), according to the Scheltens' rating scale [39]. All subjects or their legal guardians gave written informed consent before participating in the study, which was approved by the Committees of Human Research at the University of California and the VA Medical Center at San Francisco.

2.2. Data Acquisition. All scans were preformed on a 4 Tesla (Bruker/Siemens) MRI system with a single housing birdcage transmit and 8-channel receive coil. T1-weighted images were obtained using a $3 \mathrm{D}$ volumetric magnetization prepared rapid gradient echo (MPRAGE) sequence with TR/ $\mathrm{TE} / \mathrm{TI}=2300 / 3 / 950 \mathrm{~ms}$, 7 -degree flip angle, $1.0 \times 1.0$ $\times 1.0 \mathrm{~mm}^{3}$ resolution, 157 continuous sagittal slices. In addition, FLAIR (fluid attenuated inversion recovery) images with timing TR/TE/TI $=5000 / 355 / 1900 \mathrm{~ms}$ were acquired to facilitate the evaluation of WMSH. Perfusion images were acquired using a continuous arterial spin labeling (cASL) sequence [40] with a single-shot echo-planar imaging (EPI) part to map the perfusion signal. cASL-MRI was performed with $\mathrm{TR} / \mathrm{TE}=5200 / 9 \mathrm{~ms}$ with 2 -second long labeling pulses and a one-second postlabeling delay. Sixteen slices with $5 \mathrm{~mm}$ slice thickness and $1.2 \mathrm{~mm}$ interslice gap, $3.75 \times 3.75 \mathrm{~mm}^{2}$ in-plane resolution were acquired. DTI was acquired based on a dual spin-echo EPI sequence supplemented with twofold parallel imaging acceleration (GRAPPA) [41] to reduce susceptibility distortions. Other imaging parameters were $\mathrm{TR} / \mathrm{TE}=6000 / 77 \mathrm{~ms}$, field of view $256 \times 224 \mathrm{~cm}, 128 \times$ 112 matrix size, yielding $2 \times 2 \mathrm{~mm}^{2}$ in-plane resolution, 40 slices each $3 \mathrm{~mm}$ thick. One reference image $(b=0)$ and six diffusion-weighted images $\left(b=800 \mathrm{~s} / \mathrm{mm}^{2}\right.$, along 6 noncollinear directions) were acquired.

2.3. Data Analyses. The assessment of brain volume changes were performed using SPM8 software (http://www.fil.ion .ucl.ac.uk/spm/software/spm8/) based on an "optimized" VBM procedure described by Ashburner and Friston [42]. The procedure included several steps. (1) Tissue segmentation: An expectation maximization segmentation (EMS) 
TABLe 1: Demographic and clinical data summary.

\begin{tabular}{lccc}
\hline & Normal & AD & FTD \\
\hline Number of subjects & 21 & 20 & 20 \\
Age (years) & $61.9 \pm 9.6$ & $63.1 \pm 6.9$ & $60.7 \pm 9.9$ \\
Age range (years) & $33 \sim 73$ & $51 \sim 73$ & $32 \sim 74$ \\
Sex (M:F) & $11: 10$ & $11: 9$ & $13: 7$ \\
Years of Education (years) & $16.8 \pm 2.5$ & $15.7 \pm 3.0$ & $16.2 \pm 3.2$ \\
MMSE & $29.6 \pm 0.5$ & $21.9 \pm 5.6$ & $23.1 \pm 5.6$ \\
CDR & 0 & $0.8 \pm 0.3$ & $1.2 \pm 0.6$ \\
APOE- $\varepsilon 4$ (carriers: non-carriers) & $3: 17^{\mathrm{a}}$ & $14: 5^{\mathrm{a}}$ & $7: 11^{\mathrm{b}}$ \\
Age of onset (years) & NA & $56.2 \pm 5.7^{\mathrm{c}}$ & $55.1 \pm 9.9$ \\
Symptom duration (years) & NA & $3.25 \pm 1.6^{\mathrm{c}}$ & $5.3 \pm 4.9$ \\
WMSH (severe: moderate $:$ mild) & $3: 2: 16$ & $1: 4: 15$ & $2: 2: 16$ \\
\hline
\end{tabular}

a value for one subject is missing.

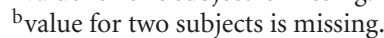

${ }^{c}$ value for 8 subjects is missing.

$\mathrm{WMSH}=$ white matter signal hyperintensities.

algorithm [43] was applied to obtain probabilistic maps of GM, WM, and CSF from the T1-weighted MRI data. (2) Spatial normalization: first, customized GM and WM prior images were created by transforming GM and WM probabilistic maps of all subjects into the standard MNI (Montreal Neurological Institute) space [42]. The segmented GM probabilistic maps in their native spaces were then spatially normalized again to the customized GM prior image using a nonlinear transformation with 16 interactions. (3) Jacobian modulation: the spatially normalized GM images were multiplied by the Jacobian determinants of the transformation (modulation) to obtain volume differences. (4) Smoothing: the modulated GM images were smoothed with an $8 \mathrm{~mm}$ full-width-at-half-maximum (FWHM) isotropic Gaussian kernel to reduce variations from misregistrations and to perform voxelwise image statistics.

The assessment of perfusion changes included the following steps. (1) Cerebral blood flow (CBF) image calculation: a perfusion weighted (PWI) image was created by pairwise subtraction of coregistered labeled from unlabeled ASL images. The PWI images were then scaled to obtain a quantitative CBF image based on a single compartment perfusion model [44]. (2) Intermodality coregistration: ALS perfusion and the corresponding T2- and T1-weighted image were coregistered using an affine alignment to establish anatomical correspondence between CBF and segmented GM images and to compute partial volume-corrected CBF in GM. (3) Partial volume correction: partial volume correction of $\mathrm{CBF}$ images was performed by rescaling $\mathrm{CBF}$ in each voxel proportionately to the GM and WM content, assuming that perfusion of white matter is only $25 \%$ of that of GM, as detailed by $\mathrm{Du}$ et al. [16]. (4) Spatial normalization: the partial volume-corrected CBF images in GM were spatially normalized to the customized GM prior image that was created from the previous processing of VBM, using the same nonlinear transformation and smoothing parameters.

The assessment of white matter integrity was performed based on DTI and computation of fractional anisotropy (FA) maps, using the dTV.II software [45] and Volume-one software package (URL: http://www.ut-radiology.umin.jp/ people/masutani/dTV.htm), supplemented by automatic image denoising and eddy-current corrections. SPM8 software was used for voxelwise analysis of the FA images as outlined in detail elsewhere [25]. In brief, the FA images in the native space underwent the following procedures. (1) Creation of a customized FA template: an averaged FA image was first created from all subjects' FA images that initially transformed to the EPI-derived MNI template in SPM. This averaged FA image was further co-registered to the WM prior image using affine alignment and manual adjustment [46] to archive a customized FA template that accurately correspond to the anatomical information in the WM prior image. (2) Spatial normalization and smoothing: the FA image of each subject in the native space was recursively normalized to the customized FA template using the same nonlinear transformation and smoothing parameters that were applied in the previous VBM procedure.

2.4. Statistics. Paired group differences were evaluated voxelby-voxel using a general linear model with diagnosis as main contrast and age, sex, and the years of education as covariates. In addition, total intracranial volume (TIV) was used as a covariate in test for volume differences and global mean perfusion was used as a covariate in test for perfusion differences. A threshold of at least 85\% GM volume fraction in voxels was applied to restrict GM volume and perfusion analyses to voxels containing predominantly GM. Similarly, a threshold of at least $80 \%$ WM volume fraction was applied to restrict FA analysis to voxels containing predominantly WM. The statistical significance for main effects of abnormalities, which was only the prestep for joint analyses, was set to an uncorrected voxel-level $P$ value of .001 .

To test if FTD and AD differ with respect to WM and GM abnormalities, we started by determining the number of voxels representing "abnormal" values for each patient and each MRI modality. This was conducted by recording voxelwise differences between an individual's image value (GM volume, GM perfusion, or WM FA) and the respective mean 
value of the control group. The difference was then normalized to the standard error of the control mean value to express abnormality as a T-score [47]. High T-scores represent high abnormalities beyond the normal ranges. Next, the number of "abnormal" voxels above a T-score threshold was recorded and normalized to the total number of GM or WM voxels to account for each patient and each modality to quantify the extent of brain abnormality. We termed the number of normalized "abnormal" voxels load. Differences in loads between $\mathrm{AD}$ and FTD patients were evaluated statistically via permutation test with 1000 -fold completely random resampling of the diagnostic labels (AD and FTD), using the R project (http://www.r-project.org/). The statistics on the loads were evaluated for T-score values ranging from -2 (indicating a mild abnormality level) to -6 (indicating a severe abnormality level). Using the permutation test, we also assessed whether the load from a specific MRI modality (e.g., WM FA) relative to another (e.g., GM loss) modality, termed conditional load, differed within and across the diagnosis groups. The level of significance for permutation tests was set at $P=.05$.

\section{Results}

3.1. Demographic Clinical Data. As shown in Table 1, there were no significant differences in age, sex, years of education, and severity of WMSH between each patient group (AD or FTD) and controls. AD and FTD patients had significant lower MMSE $(P<.001$, by ANOVA test $)$ scores compared with controls, as expected. Furthermore, AD patients had a greater proportion of APOE- $\varepsilon 4$ carriers than $\mathrm{CN}(P=.04$ by $\chi^{2}$ test $)$ but the FTD patients $\operatorname{did}$ not $\left(P=.35\right.$ by $\chi^{2}$ test) when compared to $\mathrm{CN}$. AD and FTD patients did not differ significantly with respect to age, sex, years of education, MMSE, CDR, age of onset, symptom duration, and severity of WMSH. To avoid further reductions in sample size due to the missing values, we did not perform the joint analysis including the age of onset or the APOE- $\varepsilon 4$ genotyping as covariates across all MRI modalities, although symptom duration was associated with GM volume loss within the FTD group.

3.2. Spatial Distribution of MRI Abnormalities in $A D$ and FTD Compared to CN. Figures $1(\mathrm{a})-1(\mathrm{~d})$ depict the regional distributions of GM loss (in warm color), GM hypoperfusion (in green color), and reduced WM FA (in blue color) in AD and FTD, compared to $\mathrm{CN}$, respectively, as well as the direct comparisons between $\mathrm{AD}$ and FTD, based on voxel-wise tests prior to the joint analysis. For better visualization of regional relations, the various distributions are overlaid on each other in Figure 1(d).

Compared to $\mathrm{CN}, \mathrm{AD}$ patients showed widespread GM loss in bilateral parietal and temporal lobes. The left temporoparietal lobes had the most prominent GM loss. Other regions of GM loss in $\mathrm{AD}$ included the posterior cingulate gyrus, thalamus, and bilateral occipital lobes. Compared to CN, FTD patients showed GM loss predominantly in bilateral frontal and temporal lobes. The right frontoinsular gyrus showed the most prominent GM loss. Other regions of GM loss included limbic lobes such as bilateral anterior cingulate gyrus, uncus, subcortical nuclei including the bilateral caudate and the thalamus, and the lateral parietal lobes. Comparing FTD and AD directly, patients with $\mathrm{AD}$ showed more GM loss than FTD in bilateral occipital gyrus, left precuneus whereas FTD showed more GM loss in bilateral frontal lobes, including the orbital gyrus, inferior and medial frontal gyrus, and anterior cingulate gyrus.

Regarding perfusion, $\mathrm{AD}$ patients showed reductions relative to $\mathrm{CN}$ in bilateral temporoparietal lobes, including superior temporal gyrus, precuneus and posterior cingulate gyrus. Hypoperfusion in $\mathrm{AD}$ was most pronounced in the left temporal gyrus. Compared to CN, FTD patients showed reduced perfusion in bilateral frontal lobes, including inferior, medial, and superior frontal gyrus, anterior cingulate gyrus, and thalamus. Hypoperfusion in FTD was most pronounced in the right inferior frontal gyrus. Compared to FTD, AD patients showed significant hypoperfusion in the left superior temporal gyrus, claustrum; whereas compared to AD, FTD patients had significant hypoperfusion in right superior, middle, and bilateral medial frontal gyrus.

Regarding WM FA, AD patients had FA reductions relative to CN bilaterally in WM regions in parietal, temporal, and some frontal lobe regions. The periventricular deep WM, posterior corpus callosum and the left posterior cingulum exhibited the most prominent FA reductions. In contrast, FTD patients had widespread FA reductions relative to $\mathrm{CN}$ bilaterally in frontal and temporal lobes, and the anterior corpus callosum, and bilateral anterior cingulum were prominently involved. Compared to $\mathrm{AD}$, FTD patients had lower FA values bilaterally in frontal deep WM, anterior corpus callosum and bilateral anterior cingulum, whereas $\mathrm{AD}$ patients showed no region with significantly lower FA values when compared to the FTD group.

\subsection{Differences between $A D$ and FTD in GM Volume,} Perfusion, or WM Damage. We first tested if loads differed between $\mathrm{AD}$ and FTD. Figure 2(a) displays the loads of GM loss (2A-I), GM hypoperfusion (2A-II), and WM FA (2AIII) reduction, respectively, in $\mathrm{AD}$ and FTD over a range of T-score levels (deviation from normal). The significance of differences in the loads between $\mathrm{AD}$ and FTD as a function of T-scores is plotted in Figure 2(b), and separately for each type (GM Vol, GM Perf, WM FA) of load. Note, the loads and the $P$ values are plotted on a logarithmic scale and increasing negative T-scores indicate increasing deviation from normal values. This demonstrates that there is a significantly greater load of WM FA reduction in FTD compared to AD at mild abnormality levels (up to T-scores of about -2.3) while the significance gradually vanishes at more severe abnormality levels. There is a trend $(P=.07$ to .1$)$ towards more GM loss in FTD compared to AD at moderate to severe abnormality levels (T-scores $<-4$ ). However, the load of GM hypoperfusion does not differ significantly between $\mathrm{AD}$ and FTD across the range of T-scores.

3.4. Joint Assessment of GM Volume, Perfusion, and WM FA Damages in $A D$ or FTD. Figure 3 shows the conditional loads (the load in one MRI modality relative to another) over 


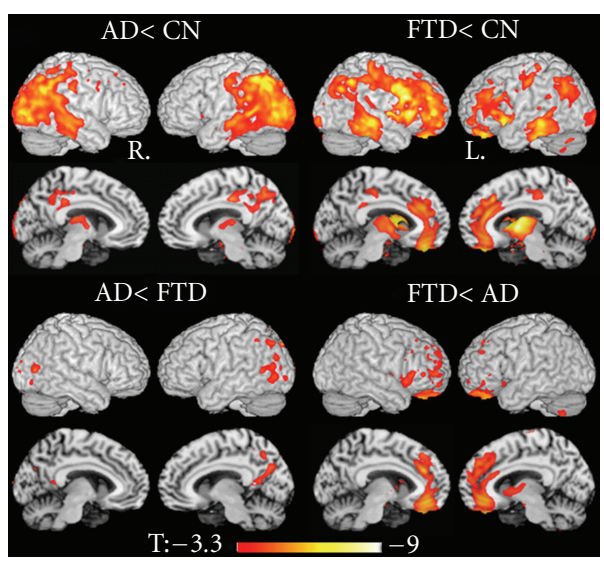

(a)

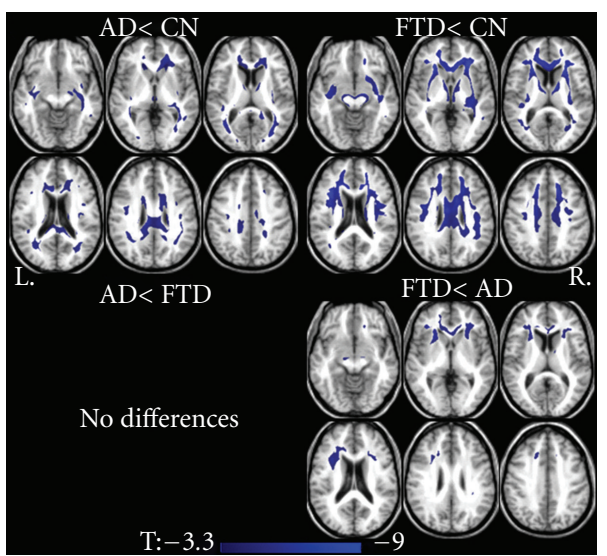

(c)

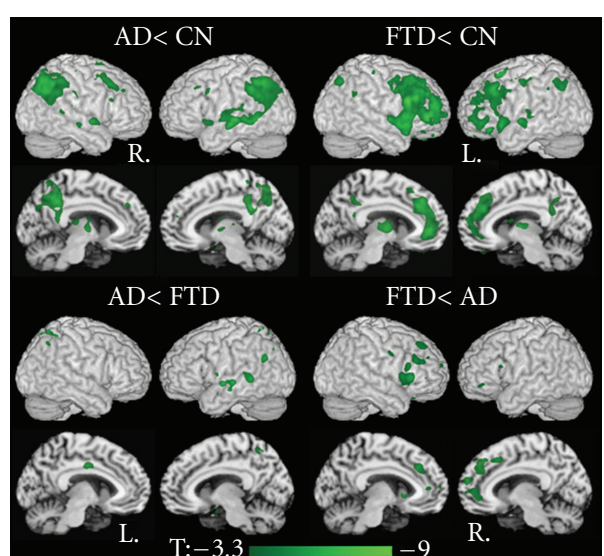

(b)

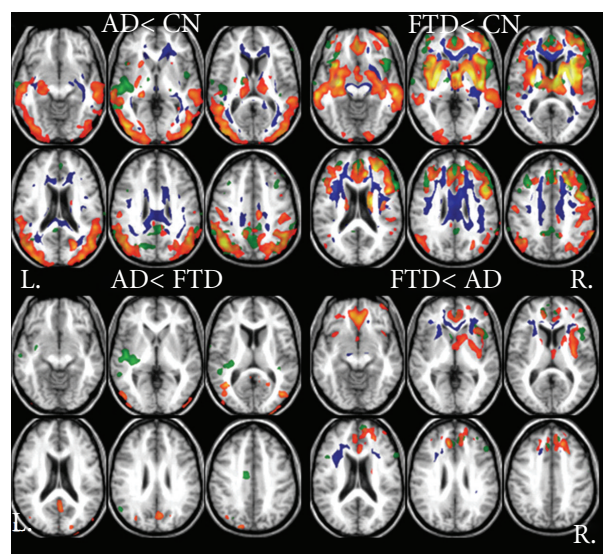

(d)

FIGURE 1: Significance maps of systematic brain abnormalities in FTD and AD patients relative to control subjects $(\mathrm{AD}<C N$ and FTD $<$ $C N$ ), and direct comparisons between $\mathrm{AD}$ and FTD (AD $<F T D$ and FTD $<A D$ ). (a) GM loss (warm color) and (b) GM hypoperfusion (green color) overlaid on a surface rendered brain template. (c) Reduced WM FA (blue color) in AD or FTD overlaid on an axial brain template. (d) Overlay of the abnormal distributions together. The significant threshold was $P_{\text {uncorrected }}<.001$ for all voxelwise tests. Color scales indicated ranges of significance (T-scores) upon the P threshold.

a range of abnormality levels (T-scores) separately for $\mathrm{AD}$ (Figure 3(a)) and for FTD (Figure 3(b)). Note, the plots in Figures 3(a) and 3(b) are on a logarithmic scale. Accordingly, a conditional load larger than 1 indicates that the load of a particular modality is greater than the load of the reference modality, whereas a value smaller than 1 indicates that the load of the reference is greater than particular modality. The extent to which differences in one load (on particular modality) relative to another (on reference modality) are above chance is depicted in Figures 3(c) and 3(d) for AD and FTD, respectively. Note again, $P$-values in Figures $3(\mathrm{c})$ and $3(\mathrm{~d})$ are plotted on a logarithmic scale of the base-10 logarithm. The loads of GM volume loss relative to GM hypoperfusion as a function of severity (T-scores) are displayed in brown lines, reduced WM FA relative to GM hypoperfusion in purple lines, and GM volume loss relative to WM FA in red lines.

Figures 3(a) and 3(c) show that in $\mathrm{AD}$, the loads of the different modalities are not significantly different compared to each other across the level of abnormalities (T-scores) levels. In FTD, by contrast (Figures 3(b) and 3(d)), the load of GM volume loss as well as the load of reduced WM FA are each significantly greater relative to the load of GM hypoperfusion at lower levels of abnormality (up to T-scores $=-4$ ). However, as abnormality increases (Tscores $<-4)$, the significance of the difference in the load of GM volume loss or WM FA reductions relative to GM hypoperfusion gradually disappears. There are no significant differences between the load of GM loss and the load of WM FA reductions across all ranges of abnormalities levels in FTD patients.

3.5. Differences between $A D$ and FTD in Joint Modalities. Finally, we also tested if AD and FTD differed with regard to their conditional loads from a particular modality relative to that from reference modality but found no significant difference between the groups $(P>.1)$ across all ranges of the abnormalities (T-score) levels. 


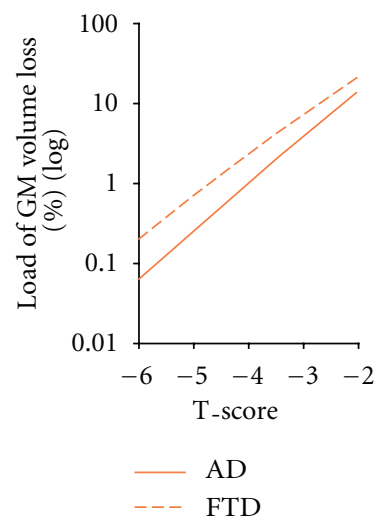

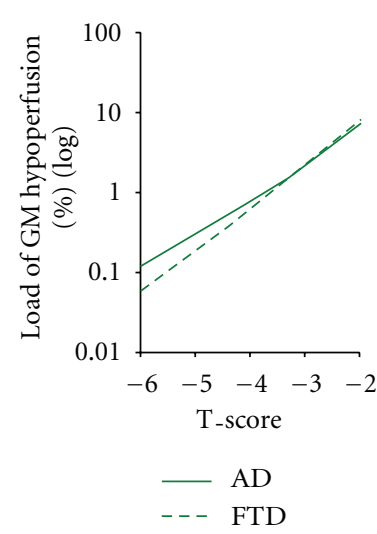

(a)

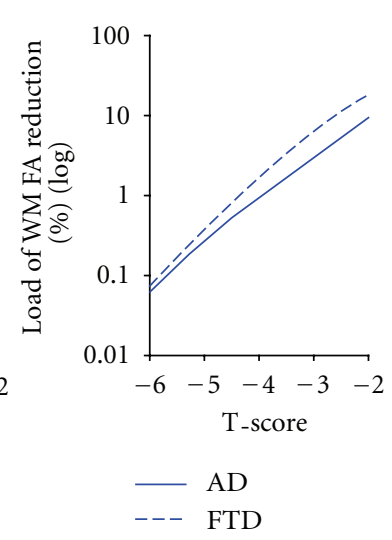

$---\quad$ FTD

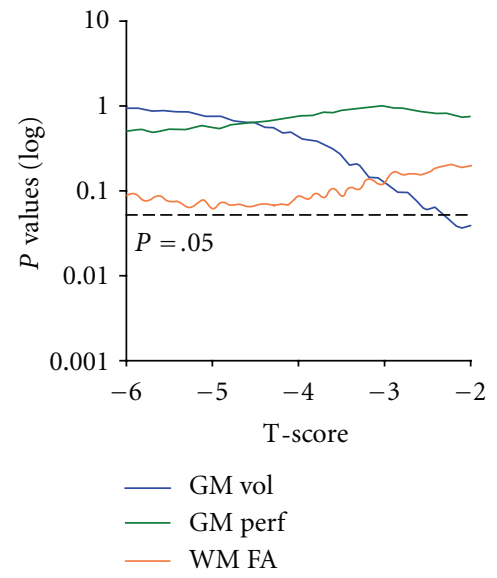

(b)

FIGURE 2: Differences in loads of GM loss (orange lines), GM hypoperfusion (green lines), and reduced WM FA (blue lines) between FTD and $\mathrm{AD}$ across a range of T-scores and significant levels. (a) Mean loads of GM loss (I), GM hypoperfusion (II), and reduced WM FA (III) along a range of abnormality levels (T-scores) in AD (solid lines) and FTD (dash lines) patients. (b) Variations in significance of the load differences between FTD and AD as a function of T-scores. Note, the vertical axis of all plots in (a, b) uses a base-10 logarithmic scale. The horizontal dotted line indicates $P=.05$ significance.

\section{Discussion}

We have two main findings. First, FTD patients exhibited more WM damage than AD patients at mild abnormality levels (i.e., small T-scores). The difference vanished gradually for more severe abnormality levels (i.e., larger T-scores). Second, FTD patients had greater GM loss and WM damage relative to GM hypoperfusion, although the differences of damage between modalities gradually vanished with increasing levels of abnormality. In contrast to FTD, AD patients had equal damage in all three modalities, irrespective of the level of abnormality. Taken together, the results suggest that FTD and AD differ in amount of WM and GM structural and functional damages, in addition to their characteristic regional patterns of brain alterations than healthy controls.

Our first main finding of greater WM damage in FTD than $\mathrm{AD}$ at mild levels of abnormality suggests that WM may be more sensitive to the pathology of FTD than to the pathology of $\mathrm{AD}$ at their early disease stages. The hallmark of FTD is tauopathies or ubiquitin immunoreactive inclusions by the presences of neuronal and glial inclusions $[48,49]$ in gray and white matter. WM pathologies in FTD have been reported with astrocytic gliosis and oligodendroglial apoptosis, which may ultimately result in axonal degeneration [5053]. A recent study [54] also reported that oligodendroglial pathology can be predominant in FTD despite severe GM damage. On the other hand, degeneration of frontostriatal networks, which is a characteristic feature of FTD [26], suggests that WM denegation in anterior brain could also be a primary FTD pathology. In contrast to FTD, the hallmark of $\mathrm{AD}$ is the deposition of amyloid plaques and neurofibrillary tangles that are associated with loss of neurons and synapses $[55,56]$. WM pathology in AD has been suggested to occur secondarily to GM pathology and may include reduction of myelin, axons, and oligodendrocytes [57, 58]. A vascular origin of WM pathology in AD has also been suggested [59]. However, WM pathologies in AD are usually considered mild and potentially reversible [60]. Interestingly, our data showed that differences in WM damage between AD and FTD disappeared at higher abnormality levels. It is possible to suggest that $\mathrm{AD}$ and FTD undergo similar WM pathologies at a severe brain damage level. One explanation is that severe WM abnormalities are an outcome of irreversible vascular damage, such as appearance of WMSH, which affects AD and FTD similarly [61]. Another possible explanation is that WM changes resulting from degeneration of corticocortical connections may inevitably occur in both AD and FTD.

In addition, we found a trend of more GM loss in FTD than $\mathrm{AD}$ at moderate to severe levels of abnormality. This observation is consistent with histopathological studies showing substantial loss of spindle neurons in the cortex in FTD but not in $\mathrm{AD}$ [62]. The finding is also in agreement with several MRI studies [29, 30, 63, 64] which compared FTD and AD directly, showing regionally greater GM loss in FTD when compared to AD but no greater GM loss in $\mathrm{AD}$ when compared to FTD. Similarly, several longitudinal studies reported greater rates of GM atrophy in FTD when compared to $\mathrm{AD}[65,66]$. However, some MRI studies [26, 67] reported greater GM loss in AD compared to FTD, though the extent of the GM losses varied regionally. The discrepancy may result from notorious difficulties to adequately match impairment severity in FTD and AD given substantial differences in symptomatology, although our current study attempted to match the severities (such as the measures of CDR, MMSE) of AD and FTD groups as closely as possible.

The second major finding that GM loss and WM damage exceed perfusion damage in FTD may be explained by loss of brain tissue other than neurons contributing to GM atrophy in FTD. Information about differential loss of various types of brain tissue may be particularly relevant in earlier 


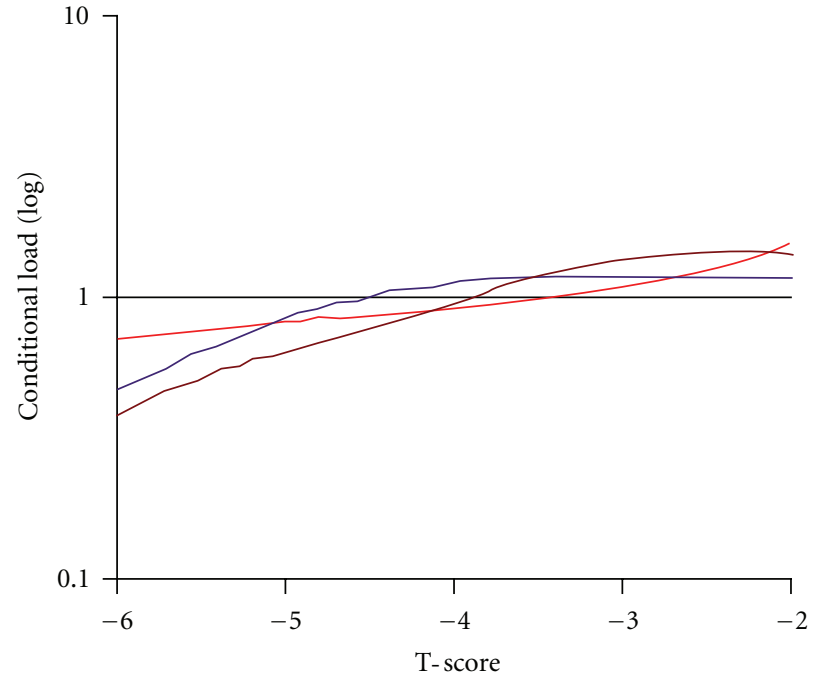

- GM vol relative to GM perf

- WM FA relative to GM perf

- GM vol relative to WM FA

(a)

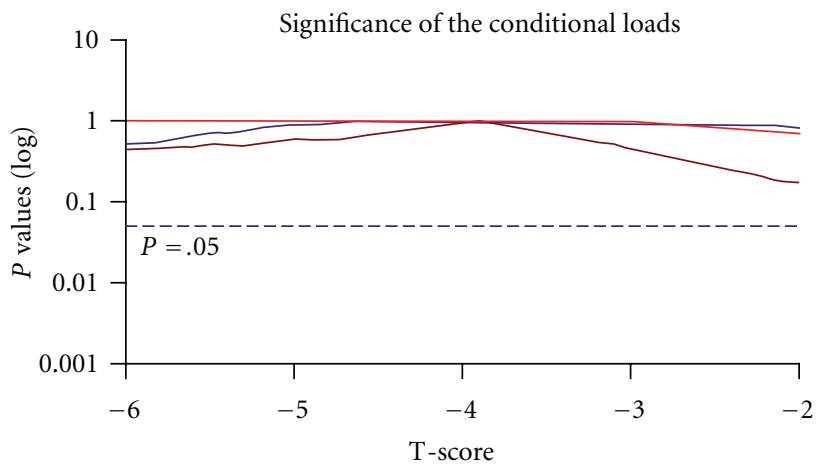

(c)

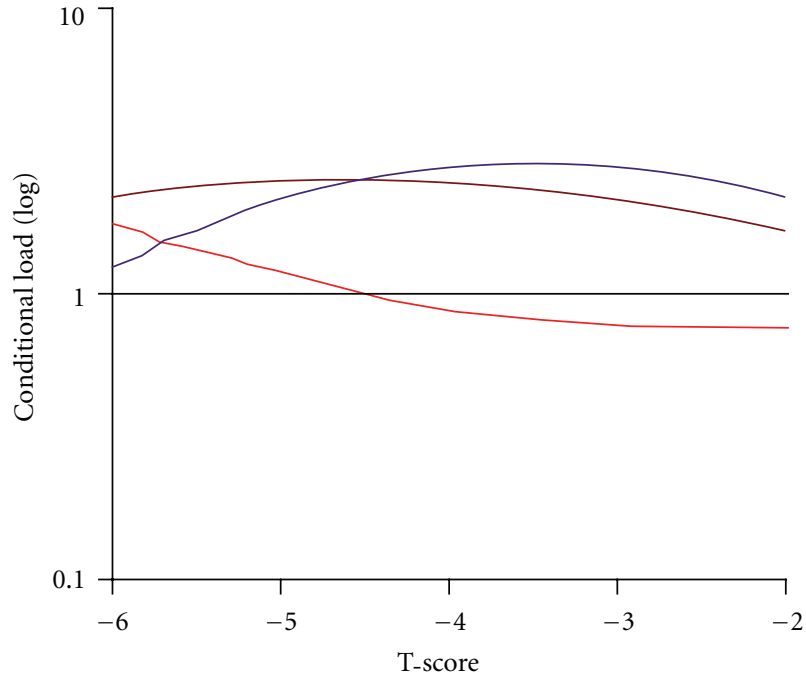

- GM vol relative to GM perf

- WM FA relative to GM perf

_ GM vol relative to WM FA

(b)

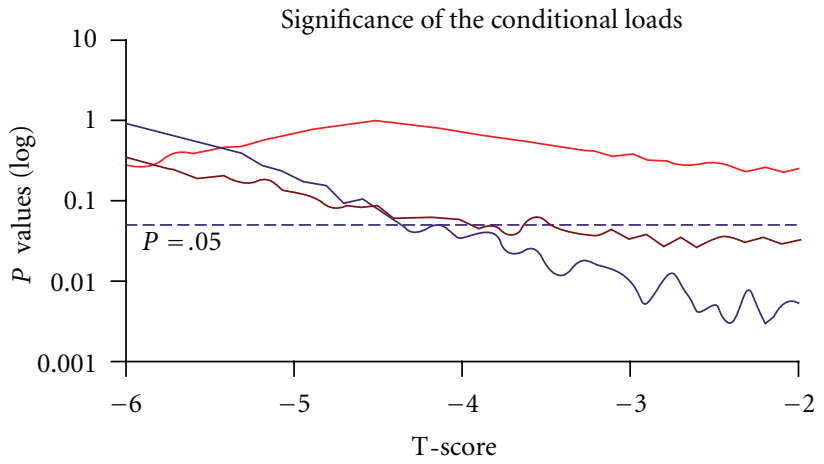

(d)

FIGURE 3: Conditional load of one modality relative to another as a function of the level of abnormality (T-score) for AD (a) and FTD (b). Note, vertical axes in (a) and (b) are plotted on a logarithmic scale (base-10) where a value larger than one indicates the load from a particular modality was higher than the load from reference modality. Conditional load lower than 1 indicated opposite relations between modalities. The extent to which differences in the conditional loads among modalities are above chance $(P<.05)$ across the level of abnormality is indicated in plot (c) for AD and in plot (d) for FTD. Note, $P$ values in (c) and (d) are also plotted on a logarithmic scale. The horizontal dash lines indicate $P=.05$ significance.

and potentially reversible stages of the disease. Our findings are supported by several histopathological studies demonstrating that the earliest cellular changes in FTD occur in astrocytes without neuron loss and that neuronal damage becomes more prominent in later stages of the disease $[50,68]$. Therefore, at mild abnormality levels of FTD, the surviving neurons may still function normally as reflected by the normal levels of perfusion. Our findings are consistent with other studies carried out in our laboratory [69] and on different cohorts of FTD and AD patients which have implied dissociation between GM atrophy and perfusion. Specifically, these studies indicated that FTD was associated with greater GM atrophy in absence of significant reduction of perfusion. These results contrast the findings in $\mathrm{AD}$, where the abnormalities across the three modalities were all similar.

Our voxelwise analysis demonstrates the regional patterns of GM loss, hypoperfusion, and reduced WM FA in this sample of $\mathrm{AD}$ and FTD patients are consistent with the disease-specific patterns that have been reported in previous MRI studies of brain structure $[14,70]$, perfusion $[5,6]$, and DTI $[25,71-73]$. Furthermore, the similarity between the regional patterns of GM and WM alterations which appear in the same lobe together for each disease, implies that WM degradation mirrors that of GM damages and is consistent with previous reports [74]. Taken together, these results can demonstrate the well-documented pathological bases of $\mathrm{AD}$ [56] and FTD [68].

The new joint analysis approach allows for the investigation of different abnormalities across multiple MRI modalities such as structural, perfusion and diffusion MRI between groups. The approach can be used to test whether groups differ with respect to a single MRI measure as well as multiple measures. The approach was augmented by nonparametric statistical tests via permutations and carried out across 
a range of T-scores to reduce measurement bias toward the various brain conditions. The concept can be expanded in principle to conduct a voxelwise joint analysis of multiple MRI measures to determine regional variations in GM loss and hypoperfusion. Other statistical methods for joint analyses of multiple image modalities such as joint independent component analysis (jICA) [75] may provide alternative solutions. The findings with multimodal MRI could potentially be useful to improve the design of AD and FTD clinical trials involving MRI. First, correlations across the MRI measures, potentially boosting sensitivity and specificity, could lead to reduced sample sizes. Second, the finding revealed that FTD presents more white matter involvement relative to $\mathrm{AD}$, thus providing a new biological feature of FTD, and could be used to relax the need to match disease severity in studies recruiting $\mathrm{AD}$ and FTD patients.

Limitations of the current study include a small sample size that was reliant on clinical diagnoses which were not sufficiently confirmed by autopsies. Therefore, confidence in the generalization of the results is limited, and potential misdiagnosis of patients may have resulted in spurious findings. Furthermore, we cannot completely rule out that other factors than disease etiology, such as genetic profiles, duration of symptoms, and cardiovascular conditions, which contributed to MRI differences between the patients and thus contaminated the findings. Second, diffusion encoding was limited to the minimum of 6 directions at the time this protocol was initiated, although it is known that many more encoding directions improve the characterization of diffusion such as fewer ambiguities in regions of crossing fibers and better spatial invariance of the noise pattern. Therefore, fiber crossings and the DTI noise pattern may potentially mimic regional differences in FA between these groups. Third, we ignored relationships between brain regions in our joint analysis and therefore under-utilized information from the multimodal MRI data. A more powerful statistical framework [76] that takes spatial relations between multivariate measures into account may provide more power. Finally, the data was artificially scaled to provide a uniform resolution for all MRI modalities, which may have induced a spatial bias as well as altered selectively the sensitivity of each modality. Other approaches that do not require a uniform resolution but can operate on variable spatial scales, such as information theoretic formalisms [77], may lead to differences in results.

In conclusion, our findings suggest that FTD and AD differ regarding their impacts on WM and GM structural and functional abnormalities, in addition to differences between their characteristic regional patterns of brain alterations. Furthermore, the joint assessment of multimodal MRI measures employed in this study has potential value to improve the differential diagnosis between FTD and AD.

\section{Acknowledgments}

This research was funded in part by National Institutes of Health Grants (P01AG19724, P50 AG23501) and a Grant from the National Center for Resource Research (P41
RR23953). This material is the result of work supported with resources and the use of facilities at the Veterans Administration Medical Center, San Francisco California. The authors thank all the participants in this study. The authors also thank Mr. Shannon Buckley and Mr. Pouria Mojabi for assistance with image processing, Mr. Philip Insel for the help with statistics, and Dr. Susanne Mueller for the advice in data analysis.

\section{References}

[1] N. S. Ryan and N. C. Fox, "Imaging biomarkers in Alzheimer's disease," Annals of the New York Academy of Sciences, vol. 1180, pp. 20-27, 2009.

[2] J. C. Baron, G. Chetelat, B. Desgranges et al., "In vivo mapping of gray matter loss with voxel-based morphometry in mild Alzheimer's disease," NeuroImage, vol. 14, no. 2, pp. 298-309, 2001.

[3] K. Ishii, H. Sasaki, A. K. Kono, N. Miyamoto, T. Fukuda, and E. Mori, "Comparison of gray matter and metabolic reduction in mild Alzheimer's disease using FDG-PET and voxel-based morphometric MR studies," European Journal of Nuclear Medicine and Molecular Imaging, vol. 32, no. 8, pp. 959-963, 2005.

[4] P. Bartenstein, S. Minoshima, C. Hirsch et al., "Quantitative assessment of cerebral blood flow in patients with Alzheimer's disease by SPECT," Journal of Nuclear Medicine, vol. 38, no. 7, pp. 1095-1101, 1997.

[5] N. A. Johnson, G. H. Jahng, M. W. Weiner et al., "Pattern of cerebral hypoperfusion in Alzheimer disease and mild cognitive impairment measured with arterial spin-labeling MR imaging: initial experience," Radiology, vol. 234, no. 3, pp. 851-859, 2005.

[6] D. C. Alsop, J. A. Detre, and M. Grossman, "Assessment of cerebral blood flow in Alzheimer's disease by spin-labeled magnetic resonance imaging," Annals of Neurology, vol. 47, no. 1, pp. 93-100, 2000.

[7] E. J. Kim, S. S. Cho, Y. Jeong et al., "Glucose metabolism in early onset versus late onset Alzheimer's disease: an SPM analysis of 120 patients," Brain, vol. 128, no. 8, pp. 1790-1801, 2005.

[8] S. Xie, J. X. Xiao, G. L. Gong et al., "Voxel-based detection of white matter abnormalities in mild Alzheimer disease," Neurology, vol. 66, no. 12, pp. 1845-1849, 2006.

[9] O. Naggara, C. Oppenheim, D. Rieu et al., "Diffusion tensor imaging in early Alzheimer's disease," Psychiatry ResearchNeuroimaging, vol. 146, no. 3, pp. 243-249, 2006.

[10] R. Stahl, O. Dietrich, S. J. Teipel, H. Hampel, M. F. Reiser, and S. O. Schoenberg, "White matter damage in Alzheimer disease and mild cognitive impairment: assessment with diffusiontensor MR imaging and parallel imaging techniques," Radiology, vol. 243, no. 2, pp. 483-492, 2007.

[11] S. J. Teipel, R. Stahl, O. Dietrich et al., "Multivariate network analysis of fiber tract integrity in Alzheimer's disease," $\mathrm{Neu}$ roImage, vol. 34, no. 3, pp. 985-995, 2007.

[12] J. Huang, R. P. Friedland, and A. P. Auchus, "Diffusion tensor imaging of normal-appearing white matter in mild cognitive impairment and early Alzheimer disease: preliminary evidence of axonal degeneration in the temporal lobe," American Journal of Neuroradiology, vol. 28, no. 10, pp. 1943-1948, 2007.

[13] L. Wang, F. C. Goldstein, E. Veledar et al., "Alterations in cortical thickness and white matter integrity in mild cognitive impairment measured by whole-brain cortical thickness 
mapping and diffusion tensor imaging," American Journal of Neuroradiology, vol. 30, no. 5, pp. 893-899, 2009.

[14] H. J. Rosen, M. L. Gorno-Tempini, W. P. Goldman et al., "Patterns of brain atrophy in frontotemporal dementia and semantic dementia," Neurology, vol. 58, no. 2, pp. 198-208, 2002.

[15] M. Grossman, C. McMillan, P. Moore et al., "What's in a name: voxel-based morphometric analyses of MRI and naming difficulty in Alzheimer's disease, frontotemporal dementia and corticobasal degeneration," Brain, vol. 127, no. 3, pp. 628-649, 2004.

[16] A. T. Du, G. H. Jahng, S. Hayasaka et al., "Hypoperfusion in frontotemporal dementia and Alzheimer disease by arterial spin labeling MRI," Neurology, vol. 67, no. 7, pp. 1215-1220, 2006.

[17] J. Diehl, T. Grimmer, A. Drzezga, M. Riemenschneider, H. Forstl, and A. Kurz, "Cerebral metabolic patterns at early stages of frontotemporal dementia and semantic dementia: A PET study," Neurobiology of Aging, vol. 25, no. 8, pp. 10511056, 2004.

[18] T. Grimmer, J. Diehl, A. Drzezga, H. Förstl, and A. Kurz, "Region-specific decline of cerebral glucose metabolism in patients with frontotemporal dementia: a prospective F-FDGPET study," Dementia and Geriatric Cognitive Disorders, vol. 18, no. 1, pp. 32-36, 2004.

[19] K. Ishii, S. Sakamoto, M. Sasaki et al., "Cerebral glucose metabolism in patients with frontotemporal dementia," Journal of Nuclear Medicine, vol. 39, no. 11, pp. 1875-1878, 1998.

[20] Y. Jeong, S. S. Cho, J. M. Park et al., "18F-FDG PET findings in frontotemporal dementia: an SPM analysis of 29 patients," Journal of Nuclear Medicine, vol. 46, no. 2, pp. 233-239, 2005.

[21] V. A. Cardenas, A. L. Boxer, L. L. Chao et al., "Deformationbased morphometry reveals brain atrophy in frontotemporal dementia," Archives of Neurology, vol. 64, no. 6, pp. 873-877, 2007.

[22] L. L. Chao, N. Schuff, E. M. Clevenger et al., "Patterns of white matter atrophy in frontotemporal lobar degeneration," Archives of Neurology, vol. 64, no. 11, pp. 1619-1624, 2007.

[23] B. Borroni, S. M. Brambati, C. Agosti et al., "Evidence of white matter changes on diffusion tensor imaging in frontotemporal dementia," Archives of Neurology, vol. 64, no. 2, pp. 246-251, 2007.

[24] E. S. Matsuo, R. W. Shin, M. L. Billingsley et al., "Biopsyderived adult human brain tau is phosphorylated at many of the same sites as Alzheimer's disease paired helical filament tau," Neuron, vol. 13, no. 4, pp. 989-1002, 1994.

[25] Y. Zhang, N. Schuff, A. T. Du et al., "White matter damage in frontotemporal dementia and Alzheimers disease measured by diffusion MRI," Brain, vol. 132, no. 9, pp. 2579-2592, 2009.

[26] G. D. Rabinovici, W. W. Seeley, E. J. Kim et al., "Distinct MRI atrophy patterns in autopsy-proven Alzheimer's disease and frontotemporal lobar degeneration," American Journal of Alzheimer's Disease and other Dementias, vol. 22, no. 6, pp. 474-488, 2007.

[27] T. Fukui and A. Kertesz, "Volumetric study of lobar atrophy in Pick complex and Alzheimer's disease," Journal of the Neurological Sciences, vol. 174, no. 2, pp. 111-121, 2000.

[28] A. R. Varma, W. Adams, J. J. Lloyd et al., "Diagnostic patterns of regional atrophy on MRI and regional cerebral blood flow change on SPECT in young onset patients with Alzheimer's disease, frontotemporal dementia and vascular dementia," Acta Neurologica Scandinavica, vol. 105, no. 4, pp. 261-269, 2002.
[29] C. Bocti, C. Rockel, P. Roy, F. Gao, and S. E. Black, “Topographical patterns of lobar atrophy in frontotemporal dementia and Alzheimer's disease," Dementia and Geriatric Cognitive Disorders, vol. 21, no. 5-6, pp. 364-372, 2006.

[30] T. Kanda, K. Ishii, T. Uemura et al., "Comparison of grey matter and metabolic reductions in frontotemporal dementia using FDG-PET and voxel-based morphometric MR studies," European Journal of Nuclear Medicine and Molecular Imaging, vol. 35, no. 12, pp. 2227-2234, 2008.

[31] C. Tranfaglia, B. Palumbo, D. Siepi, H. Sinzinger, and L. Parnetti, "Semi-quantitative analysis of perfusion of Brodmann areas in the differential diagnosis of cognitive impairment in Alzheimer's disease, fronto-temporal dementia and mild cognitive impairment," Hellenic Journal of Nuclear Medicine, vol. 12, no. 2, pp. 110-195, 2009.

[32] A. Varrone, S. Pappata, C. Caraco et al., "Voxel-based comparison of rCBF SPET images in frontotemporal dementia and Alzheimer's disease highlights the involvement of different cortical networks," European Journal of Nuclear Medicine, vol. 29, no. 11, pp. 1447-1454, 2002.

[33] C. Luckhaus, M. Janner, M. Cohnen et al., "A novel MRIbiomarker candidate for Alzheimer's disease composed of regional brain volume and perfusion variables," European Journal of Neurology, vol. 17, no. 12, pp. 1437-1444, 2010.

[34] K. B. Walhovd, A. M. Fjell, I. Amlien et al., "Multimodal imaging in mild cognitive impairment: metabolism, morphometry and diffusion of the temporal-parietal memory network," NeuroImage, vol. 45, no. 1, pp. 215-223, 2009.

[35] M. F. Folstein, S. E. Folstein, and P. R. McHugh, “'Mini mental state': a practical method for grading the cognitive state of patients for the clinician," Journal of Psychiatric Research, vol. 12, no. 3, pp. 189-198, 1975.

[36] D. Neary, J. S. Snowden, L. Gustafson et al., "Frontotemporal lobar degeneration: a consensus on clinical diagnostic criteria," Neurology, vol. 51, no. 6, pp. 1546-1554, 1998.

[37] G. McKhann, D. Drachman, M. Folstein, R. Katzman, D. Price, and E. M. Stadlan, "Clinical diagnosis of Alzheimer's disease: report of the NINCDS- ADRDA Work Group under the auspices of Department of Health and Human Services Task Force on Alzheimer's Disease," Neurology, vol. 34, no. 7, pp. 939-944, 1984.

[38] J. C. Morris, "The Clinical Dementia Rating (CDR): current version and scoring rules," Neurology, vol. 43, no. 11, pp. 24122414, 1993.

[39] P. Scheltens, F. Barkhof, D. Leys et al., "A semiquantitative rating scale for the assessment of signal hyperintensities on magnetic resonance imaging," Journal of the Neurological Sciences, vol. 114, no. 1, pp. 7-12, 1993.

[40] J. A. Detre, J. S. Leigh, D. S. Williams, and A. P. Koretsky, "Perfusion imaging," Magnetic Resonance in Medicine, vol. 23, no. 1, pp. 37-45, 1992.

[41] M. A. Griswold, P. M. Jakob, R. M. Heidemann et al., "Generalized autocalibrating partially parallel acquisitions (GRAPPA)," Magnetic Resonance in Medicine, vol. 47, no. 6, pp. 1202-1210, 2002.

[42] J. Ashburner and K. J. Friston, "Voxel-based morphometrythe methods," NeuroImage, vol. 11, no. 6 I, pp. 805-821, 2000.

[43] K. Van Leemput, F. Maes, D. Vandermeulen, A. Colchester, and P. Suetens, "Automated segmentation of multiple sclerosis lesions by model outlier detection," IEEE Transactions on Medical Imaging, vol. 20, no. 8, pp. 677-688, 2001.

[44] J. Wang, Y. Zhang, R. L. Wolf, A. C. Roc, D. C. Alsop, and J. A. Detre, "Amplitude-modulated continuous arterial 
spin-labeling 3.0-T perfusion MR imaging with a single coil: feasibility study," Radiology, vol. 235, no. 1, pp. 218-228, 2005.

[45] Y. Masutani, S. Aoki, O. Abe, N. Hayashi, and K. Otomo, "MR diffusion tensor imaging: recent advance and new techniques for diffusion tensor visualization," European Journal of Radiology, vol. 46, no. 1, pp. 53-66, 2003.

[46] W. Zhan, Y. Zhang, S. G. Mueller et al., "Characterization of white matter degeneration in elderly subjects by magnetic resonance diffusion and FLAIR imaging correlation," NeuroImage, vol. 47, supplement 2, pp. T58-T65, 2009.

[47] M. Signorini, E. Paulesu, K. Friston et al., "Rapid assessment of regional cerebral metabolic abnormalities in single subjects with quantitative and nonquantitative [18F]FDG PET: a clinical validation of statistical parametric mapping," NeuroImage, vol. 9, no. 1, pp. 63-80, 1999.

[48] G. M. McKhann, M. S. Albert, M. Grossman, B. Miller, D. Dickson, and J. Q. Trojanowski, "Clinical and pathological diagnosis of frontotemporal dementia: report of the work group on frontotemporal dementia and Pick's disease," Archives of Neurology, vol. 58, no. 11, pp. 1803-1809, 2001.

[49] J. Q. Trojanowski, K. Duff, H. Fillit et al., "New directions for frontotemporal dementia drug discovery," Alzheimer's and Dementia, vol. 4, no. 2, pp. 89-93, 2008.

[50] M. Broe, J. Kril, and G. M. Halliday, "Astrocytic degeneration relates to the severity of disease in frontotemporal dementia," Brain, vol. 127, no. 10, pp. 2214-2220, 2004.

[51] J. M. Powers, N. P. Byrne, M. Ito et al., "A novel leukoencephalopathy associated with tau deposits primarily in white matter glia," Acta Neuropathologica, vol. 106, no. 2, pp. 181187, 2003.

[52] E. Englund and A. Brun, "Frontal lobe degeneration of nonAlzheimer type IV: white matter changes," Archives of Gerontology and Geriatrics, vol. 6, no. 3, pp. 235-243, 1987.

[53] E. M. Larsson, E. Englund, M. Sjobeck, J. Latt, and S. Brockstedt, "MRI with diffusion tensor imaging post-mortem at 3.0 T in a patient with frontotemporal dementia," Dementia and Geriatric Cognitive Disorders, vol. 17, no. 4, pp. 316-319, 2004.

[54] G. G. Kovacs, K. Majtenyi, S. Spina et al., "White matter tauopathy with globular glial inclusions: a distinct sporadic frontotemporal lobar segeneration," Journal of Neuropathology and Experimental Neurology, vol. 67, no. 10, pp. 963-975, 2008.

[55] M. E. Calhoun, P. Burgermeister, A. L. Phinney et al., "Neuronal overexpression of mutant amyloid precursor protein results in prominent deposition of cerebrovascular amyloid," Proceedings of the National Academy of Sciences of the United States of America, vol. 96, no. 24, pp. 14088-14093, 1999.

[56] H. Braak and E. Braak, "Staging of Alzheimer's disease-related neurofibrillary changes," Neurobiology of Aging, vol. 16, no. 3, pp. 271-278, 1995.

[57] M. Bozzali, A. Falini, M. Franceschi et al., "White matter damage in Alzheimer's disease assessed in vivo using diffusion tensor magnetic resonance imaging," Journal of Neurology Neurosurgery and Psychiatry, vol. 72, no. 6, pp. 742-746, 2002.

[58] H. Hanyu, H. Sakurai, T. Iwamoto, M. Takasaki, H. Shindo, and K. Abe, "Diffusion-weighted MR imaging of the hippocampus and temporal white matter in Alzheimer's disease," Journal of the Neurological Sciences, vol. 156, no. 2, pp. 195200, 1998.

[59] E. Englund, "Neuropathology of white matter changes in Alzheimer's disease and vascular dementia," Dementia and
Geriatric Cognitive Disorders, vol. 9, supplement 1, pp. 6-12, 1998.

[60] C. M. Filley and B. K. Kleinschmidt-DeMasters, "Toxic leukoencephalopathy," The New England Journal of Medicine, vol. 345, no. 6, pp. 425-432, 2001.

[61] A. R. Varma, R. Laitt, J. J. Lloyd et al., "Diagnostic value of high signal abnormalities on T2 weighted MRI in the differentiation of Alzheimer's, frontotemporal and vascular dementias," Acta Neurologica Scandinavica, vol. 105, no. 5, pp. 355-364, 2002.

[62] W. W. Seeley, D. A. Carlin, J. M. Allman et al., "Early frontotemporal dementia targets neurons unique to apes and humans," Annals of Neurology, vol. 60, no. 6, pp. 660-667, 2006.

[63] H. Kitagaki, E. Mori, S. Yamaji et al., "Frontotemporal dementia and Alzheimer disease: evaluation of cortical atrophy with automated hemispheric surface display generated with MR images," Radiology, vol. 208, no. 2, pp. 431-439, 1998.

[64] B. B. Avants, P. A. Cook, L. Ungar, J. C. Gee, and M. Grossman, "Dementia induces correlated reductions in white matter integrity and cortical thickness: a multivariate neuroimaging study with sparse canonical correlation analysis," NeuroImage, vol. 50, no. 3, pp. 1004-1016, 2010.

[65] J. L. Whitwell, C. R. Jack Jr., V. S. Pankratz et al., "Rates of brain atrophy over time in autopsy-proven frontotemporal dementia and Alzheimer disease," NeuroImage, vol. 39, no. 3, pp. 1034-1040, 2008.

[66] D. Chan, N. C. Fox, R. Jenkins, R. I. Scahill, W. R. Crum, and M. N. Rossor, "Rates of global and regional cerebral atrophy in $\mathrm{AD}$ and frontotemporal dementia," Neurology, vol. 57, no. 10, pp. 1756-1763, 2001.

[67] C. Davatzikos, S. M. Resnick, X. Wu, P. Parmpi, and C. M. Clark, "Individual patient diagnosis of AD and FTD via highdimensional pattern classification of MRI," NeuroImage, vol. 41, no. 4, pp. 1220-1227, 2008.

[68] C. Kersaitis, G. M. Halliday, and J. J. Kril, "Regional and cellular pathology in frontotemporal dementia: relationship to stage of disease in cases with and without Pick bodies," Acta Neuropathologica, vol. 108, no. 6, pp. 515-523, 2004.

[69] S. Shimizu, Y. Zhang, J. Laxamana et al., "Concordance and discordance between brain perfusion and atrophy in frontotemporal dementia," Brain Imaging and Behavior, vol. 4, no. 1, pp. 46-54, 2010.

[70] J. L. Whitwell, K. A. Josephs, M. N. Rossor et al., "Magnetic resonance imaging signatures of tissue pathology in frontotemporal dementia," Archives of Neurology, vol. 62, no. 9, pp. 1402-1408, 2005.

[71] S. Takahashi, H. Yonezawa, J. Takahashi, M. Kudo, T. Inoue, and $\mathrm{H}$. Tohgi, "Selective reduction of diffusion anisotropy in white matter of Alzheimer disease brains measured by 3.0 Tesla magnetic resonance imaging," Neuroscience Letters, vol. 332, no. 1, pp. 45-48, 2002.

[72] A. Fellgiebel, M. J. Muller, P. Wille et al., "Color-coded diffusion-tensor-imaging of posterior cingulate fiber tracts in mild cognitive impairment," Neurobiology of Aging, vol. 26, no. 8, pp. 1193-1198, 2005.

[73] D. H. Salat, D. S. Tuch, A. J. W. van der Kouwe et al., "White matter pathology isolates the hippocampal formation in Alzheimer's disease," Neurobiology of Aging, vol. 31, no. 2, pp. 244-256, 2010.

[74] G. Bartzokis, P. H. Lu, and J. Mintz, "Human brain myelination and amyloid beta deposition in Alzheimer's disease," Alzheimer's and Dementia, vol. 3, no. 2, pp. 122-125, 2007. 
[75] K. A. Celone, V. D. Calhoun, B. C. Dickerson et al., "Alterations in memory networks in mild cognitive impairment and Alzheimer's disease: an independent component analysis," Journal of Neuroscience, vol. 26, no. 40, pp. 10222-10231, 2006.

[76] K. J. Worsley, J. E. Taylor, F. Tomaiuolo, and J. Lerch, "Unified univariate and multivariate random field theory," NeuroImage, vol. 23, supplement 1, pp. S189-S195, 2004.

[77] K. Young, A. T. Du, J. Kramer et al., "Patterns of structural complexity in Alzheimer's disease and frontotemporal dementia," Human Brain Mapping, vol. 30, no. 5, pp. 1667-1677, 2009. 


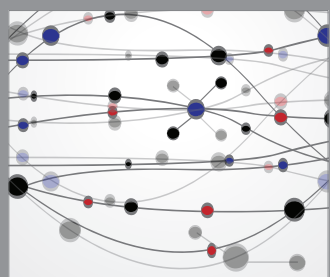

The Scientific World Journal
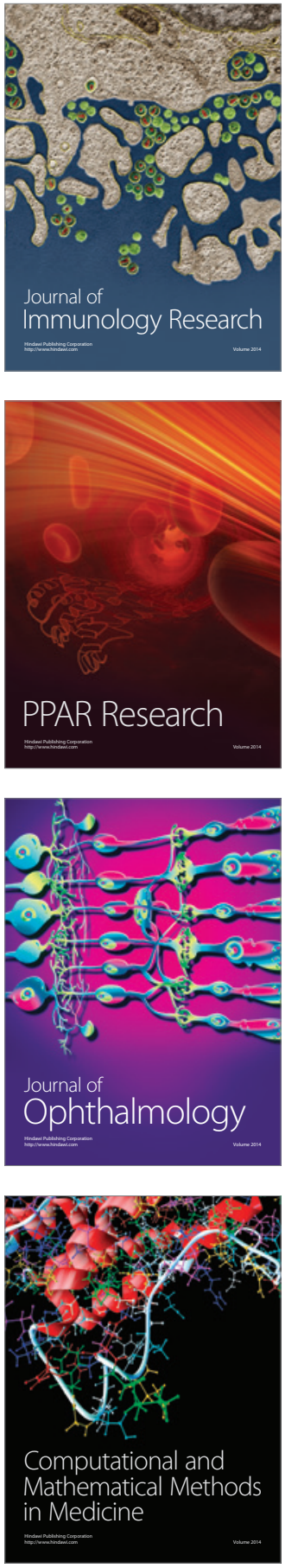

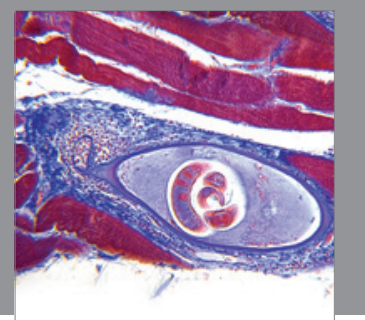

Gastroenterology

Research and Practice
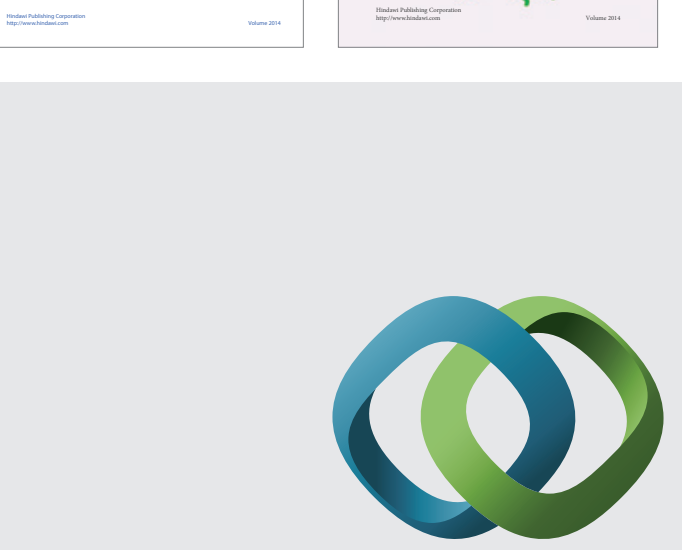

\section{Hindawi}

Submit your manuscripts at

http://www.hindawi.com
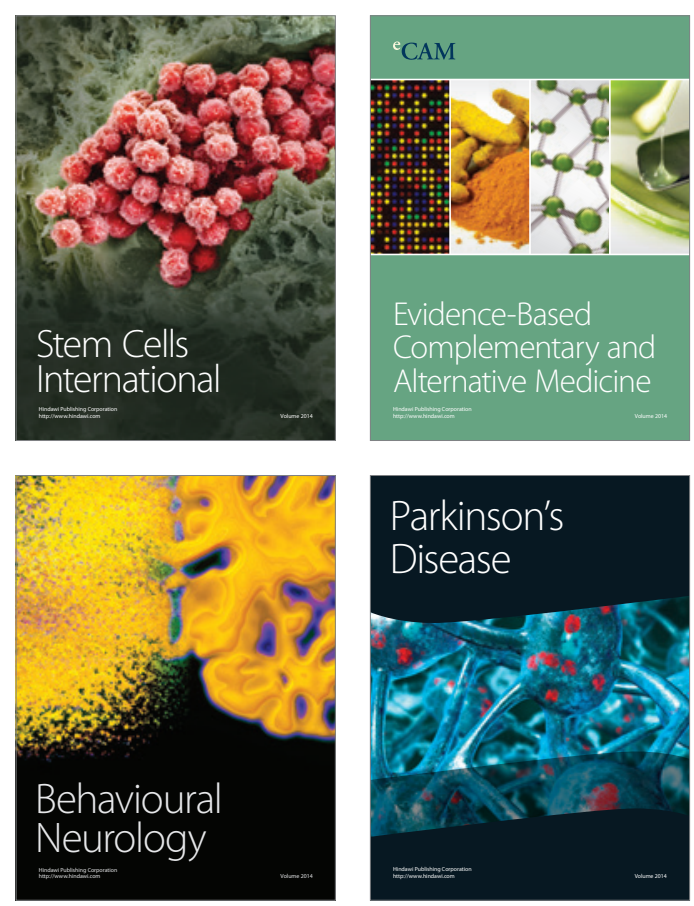

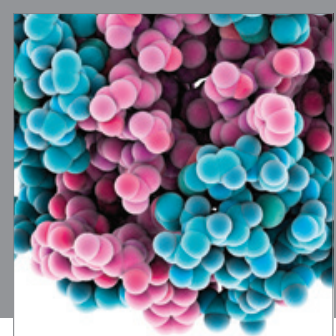

Journal of
Diabetes Research

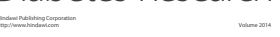

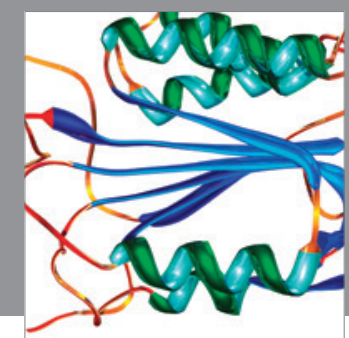

Disease Markers
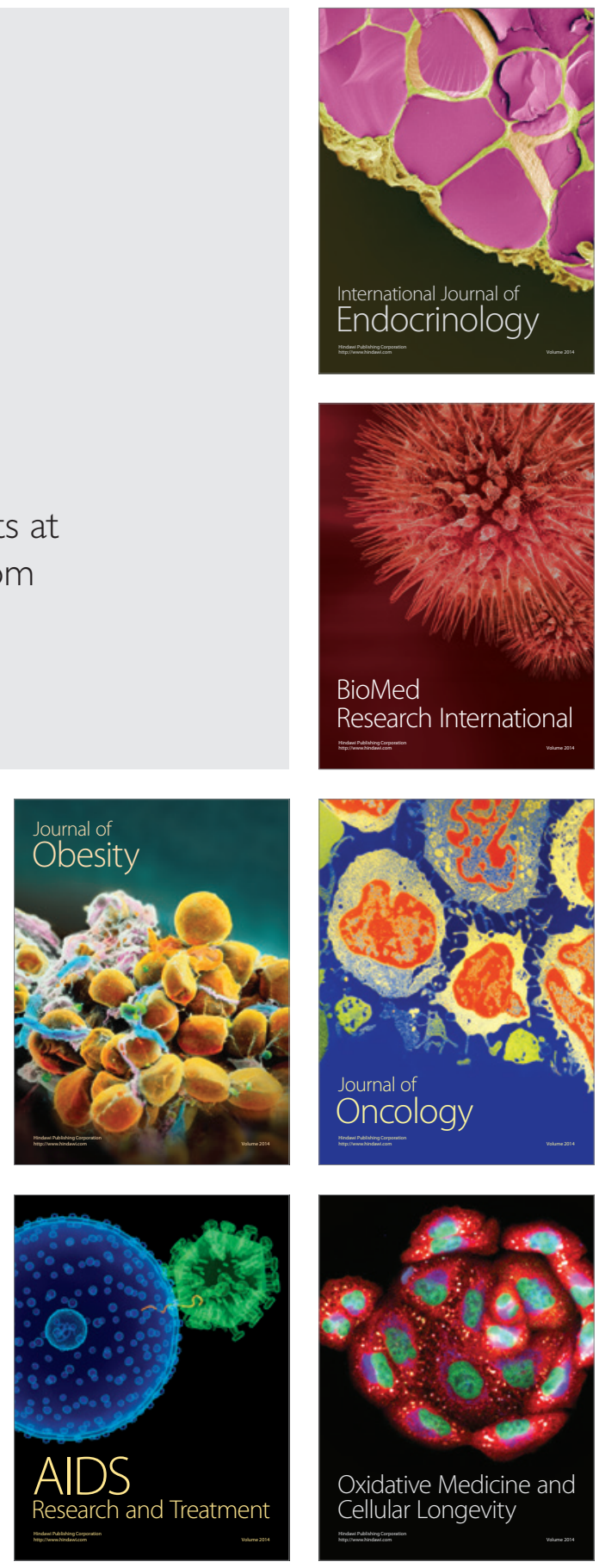